ARTICLE

https://doi.org/10.1038/s41467-019-12992-y

\title{
Polymorphic cobalt diselenide as extremely stable electrocatalyst in acidic media via a phase-mixing strategy
}

\author{
Xiao-Long Zhang ${ }^{1,6}$, Shao-Jin Hu${ }^{2,6}$, Ya-Rong Zheng (10 1,6, Rui Wu' ${ }^{1}$ Fei-Yue Gao ${ }^{1}$, Peng-Peng Yang ${ }^{1}$, \\ Zhuang-Zhuang Niu', Chao Gu${ }^{1}$, Xingxing $\mathrm{Yu}^{1}$, Xu-Sheng Zheng ${ }^{3}$, Cheng Ma ${ }^{4}$, Xiao Zheng (1) ${ }^{2}$, Jun-Fa Zhu (i) ${ }^{3}$, \\ Min-Rui Gao (1) ${ }^{1 \star} \&$ Shu-Hong Yu (i) ${ }^{1,5 *}$
}

Many platinum group metal-free inorganic catalysts have demonstrated high intrinsic activity for diverse important electrode reactions, but their practical use often suffers from undesirable structural degradation and hence poor stability, especially in acidic media. We report here an alkali-heating synthesis to achieve phase-mixed cobalt diselenide material with nearly homogeneous distribution of cubic and orthorhombic phases. Using water electroreduction as a model reaction, we observe that the phase-mixed cobalt diselenide reaches the current density of 10 milliamperes per square centimeter at overpotential of mere 124 millivolts in acidic electrolyte. The catalyst shows no sign of deactivation after more than $400 \mathrm{~h}$ of continuous operation and the polarization curve is well retained after 50,000 potential cycles. Experimental and computational investigations uncover a boosted covalency between Co and Se atoms resulting from the phase mixture, which substantially enhances the lattice robustness and thereby the material stability. The findings provide promising design strategy for long-lived catalysts in acid through crystal phase engineering.

\footnotetext{
${ }^{1}$ Division of Nanomaterials \& Chemistry, Hefei National Laboratory for Physical Sciences at the Microscale, CAS Center for Excellence in Nanoscience, Hefei Science Center of CAS, Collaborative Innovation Center of Suzhou Nano Science and Technology, Department of Chemistry, University of Science and Technology of China, 230026 Hefei, China. ${ }^{2}$ Division of Theoretical and Computational Sciences, Hefei National Laboratory for Physical Sciences at the Microscale, Department of Chemical Physics, University of Science and Technology of China, 230026 Hefei, Anhui, P. R. China. ${ }^{3}$ National Synchrotron Radiation Laboratory, University of Science and Technology of China, 230029 Hefei, P. R. China. ${ }^{4}$ Department of Materials Science and Engineering, University of Science and Technology of China, 230026 Hefei, China. ${ }^{5}$ Dalian National Laboratory for Clean Energy, 116023 Dalian, China. ${ }^{6}$ These authors contributed equally: Xiao-Long Zhang, Shao-Jin Hu, Ya-Rong Zheng. *email: mgao@ustc.edu.cn; shyu@ustc.edu.cn
} 
C lean-energy technologies such as fuel cells and electrolyzers require even more active and stable electrocatalysts that accelerate the multi-proton/multi-electron-involved electrode reactions ${ }^{1,2}$. Previous studies have shown diverse approaches to boosting catalytic activity, such as chemical doping 3,4 , strain ${ }^{5,6}$ and defect engineering 7 , alloying ${ }^{8-10}$ and dealloying ${ }^{11}$. Moreover, several activity descriptors that guide the design of energetic catalysts-including the $d$-band center associated with oxygen reduction reaction (ORR) ${ }^{12}$ and the $e_{g}$ filling associated with oxygen evolution reaction (OER) ${ }^{13}$, as well as the $\Delta G_{\mathrm{H}}$ binding energy related with hydrogen evolution reaction (HER $)^{14}$-have been proposed, advancing the development of new electrocatalysts. However, the degradation of many active catalysts in harsh electrochemical environments, such as $\mathrm{RuO}_{2}$ in OER process ${ }^{15}$ and $\mathrm{Pt}$ alloys in ORR process ${ }^{10}$, has remained a major challenge that limits the device efficiency and cost effectiveness.

The stability of electrocatalysts could be governed by multiparameters, including the bulk Pourbaix thermodynamics, dissolution potential, and solution $\mathrm{pH}$. In practical electrochemical process, catalysts may suffer from poisoning by impurity gases (e.g., CO $)^{16}$ and corrosion by in situ formed radicals (e.g., $\left.\cdot \mathrm{OH}\right)^{17}$, as well as particle coarsening ${ }^{18}$, which cause severe deactivation. Prior efforts suggest that the stability can be improved in various ways-for example, by catalyst encapsulation ${ }^{19,20}$, compositional modulation $10,21,22$, or coupling with catalyst supports ${ }^{23}$. Despite improvements in catalyst stability with these strategies, most operational stability studies have been performed on Pt-group catalysts ${ }^{10,20-23}$. As with emerging nonprecious metal-based catalysts, their long-term stability, particularly in acidic electrolyte, is unsatisfactory. This consequently leads to the problem of using nonprecious catalysts in polymer electrolyte membranebased electrochemical devices, which need low $\mathrm{pH}$ environment for operation.

Here we describe a way to improve the stability of polymorphic cobalt diselenide $\left(\mathrm{CoSe}_{2}\right)$ catalyst through homogeneous phase mixing between cubic and orthorhombic phases. In acidic electrolyte $\left(0.5 \mathrm{M} \mathrm{H}_{2} \mathrm{SO}_{4}, \mathrm{pH}=0\right)$, the phase-mixed $\mathrm{CoSe}_{2}(\mathrm{~m}$ $\mathrm{CoSe}_{2}$ ) requires an overpotential of $124 \mathrm{mV}$ at $10 \mathrm{~mA} \mathrm{~cm}^{-2}$, which was perfectly retained for over $400 \mathrm{~h}$ of continuous operation, consistent with the observation from accelerated stability tests after 50,000 electrochemical cycles. This remarkable HER stability of phase-mixed $\mathrm{CoSe}_{2}$ at low $\mathrm{pH}$ can be ascribed to the greater covalency between $\mathrm{Co}$ and $\mathrm{Se}$ atoms, resulting in lattice configuration with enhanced robustness. We expect that such phase mixing method could be applicable to other polymorphic materials for designing new electrocatalysts with better catalytic performances.

\section{Results}

Synthesis and characterization of $\mathbf{m}-\mathrm{CoSe}_{2}$. Ultrathin cubic $\mathrm{CoSe}_{2}\left(\mathrm{c}-\mathrm{CoSe}_{2}\right)$ nanobelts were synthesized as precursors using the method described previously ${ }^{24}$ (Supplementary Fig. 1). We recently reported on the complete structural phase transition of c$\mathrm{CoSe}_{2}$ to orthorhombic $\mathrm{CoSe}_{2}\left(\mathrm{o}-\mathrm{CoSe}_{2}\right)$ induced by phosphorus doping at a high temperature of $400{ }^{\circ} \mathrm{C}$ (ref. ${ }^{25}$ ). In this study, we develop an alkali-heating approach to produce $\mathrm{m}-\mathrm{CoSe}_{2}$ with nearly homogeneous distribution of cubic and orthorhombic phases (Supplementary Fig. 2). Briefly, the as-made c-CoSe powder was placed in $5 \mathrm{M} \mathrm{KOH}$ solution and heated at $200{ }^{\circ} \mathrm{C}$ for $12 \mathrm{~h}$. This harsh condition causes the leaching of $\mathrm{Co}$ and $\mathrm{Se}$ at defective sites of $\mathrm{c}-\mathrm{CoSe}_{2}$, leaving atomic vacancies (Fig. 1a). Buseck et al. ${ }^{26}$ proposed that lattice defects in cubic pyrites could offer sites to drive its transition to orthorhombic marcasite. Because pyrite $\mathrm{CoSe}_{2}$ (100) shows almost the same atomic arrangement with marcasite $\mathrm{CoSe}_{2}$ (101) (Supplementary Fig. 3), it permits locally epitaxial growth of $\mathrm{o}-\mathrm{CoSe}_{2}$ at these vacancy defects. Density functional theory (DFT) calculations in Fig. 1b reveal that the $c-\mathrm{CoSe}_{2}(100) \| \mathrm{o}-\mathrm{CoSe}_{2}$ (101) interfacial energy is smaller than the surface energies of $\mathrm{c}-\mathrm{CoSe}_{2}(100)$ and $\mathrm{o}-\mathrm{CoSe}_{2}$ (101), suggesting that the epitaxial growth of o-CoSe $e_{2}$ is thermodynamically favored. We thus conclude that the in situ formed atomic vacancies mediated the formation of $\mathrm{o}-\mathrm{CoSe}_{2}$ phase, resulting in unusual $\mathrm{m}-\mathrm{CoSe}_{2}$ (Fig. 1a), which is analogous to the Se vacancies induced 1T-to-1H transition in $\mathrm{PtSe}_{2}$ film reported recently ${ }^{27}$.

Our series of control experiments show that alkali and temperature are critical to trigger structural phase transition in c-CoSe $e_{2}$ (Supplementary Figs. 4-13). Without alkali or aging c$\mathrm{CoSe}_{2}$ in $5 \mathrm{M} \mathrm{KOH}$ at room temperature, the Co and Se leaching from c-CoSe $e_{2}$ was kinetically limited, as confirmed by inductively coupled plasma atomic emission spectroscopy (ICP-AES; Fig. 1c). Consequently, it lacks the needed vacancy defects in $\mathrm{c}-\mathrm{CoSe}_{2}$ where the phase transition occurs. The above experiments also uncover that heating $\mathrm{c}-\mathrm{CoSe}_{2}$ at $200^{\circ} \mathrm{C}$ for $12 \mathrm{~h}$ in $5 \mathrm{M} \mathrm{KOH}$ would result in the optimal m-CoSe $e_{2}$ (Supplementary Figs. 8-13). Alkali-heating treatment has been widely applied on carbon materials ${ }^{28}$ (e.g., graphene $\mathrm{e}^{29}$ and carbon nanotubes ${ }^{30}$ ) to improve the porosity, electrochemical performance, and corrosion resistance. We note that no collapse of $\mathrm{c}-\mathrm{CoSe}_{2}$ structure under above harsh conditions is unexpected, which implies the remarkable robustness of the achieved $\mathrm{m}-\mathrm{CoSe}_{2}$.

Scanning transmission electron microscopy (STEM) image in Fig. 2a shows surface scratches on $\mathrm{m}-\mathrm{CoSe}_{2}$ after the alkaliheating process, consistent with the observations from scanning electron microscopy (SEM; Supplementary Fig. 14a) and transmission electron microscopy (TEM; Supplementary Fig. 14b) images. Atomic force microscopy (Supplementary Fig. 15a) and high-angle annular dark field (HAADF; Supplementary Fig. 15b) images clearly reveal that the alkali-heating treatment etches c$\mathrm{CoSe}_{2}$ precursors and has created slit nanopores with sizes ranging from 1.4 to $7.7 \mathrm{~nm}$ (Supplementary Fig. 16). These nanopores were formed owing to the Co and Se leaching, which makes atomic level interfaces with high surface free energy where the rearrangement of Co and Se could happen. Energy-dispersive $\mathrm{X}$-ray spectrum (EDX) elemental mapping in Fig. $2 \mathrm{~b}$ shows a uniform spatial distribution of $\mathrm{Co}$ and $\mathrm{Se}$ in $\mathrm{m}-\mathrm{CoSe}_{2}$ sample, and its overall Co:Se ratio remains about 1:2 based on EDX analysis (Supplementary Fig. 17).

Figure $2 c$ shows the inverse fast Fourier transform (FFT) image of m-CoSe $e_{2}$ from a typical HAADF-STEM image (Supplementary Fig. 18a), which clearly displays the distribution the $\mathrm{c}-\mathrm{CoSe}_{2}$ (green) and $\mathrm{o}-\mathrm{CoSe}_{2}$ (red). Inverse FFT images from other regions of $\mathrm{m}-\mathrm{CoSe}_{2}$ nanobelts further confirm the unique phasemixed microstructure (Supplementary Fig. 19). Atomicresolution HAADF-STEM image in Fig. 2d reveals that the c$\mathrm{CoSe}_{2}$ and $\mathrm{o}-\mathrm{CoSe}_{2}$ phases can be distinguished from their different crystalline structures, where the FFT pattern (inset in Fig. 2d) can be interpreted as two sets of patterns from the [111] zone axis of $\mathrm{c}-\mathrm{CoSe}_{2}$ and [100] zone axis of o-CoSe $e_{2}$. Figure 2e, f shows HAADF-STEM images taken at higher magnifications from the areas marked by the red and yellow dashed squares. The c-CoSe $e_{2}$ lattice exhibits a honeycomb structure (Fig. 2e), which is unambiguously discriminated from the o- $\mathrm{CoSe}_{2}$ with parallel line lattices (Fig. 2f). The corresponding FFT patterns demonstrate cubic and orthorhombic phases at the two regions (Insets of Fig. 2e, f), consistent with our FFT simulation results (Supplementary Fig. 20). The co-existed two phases are further visualized by high-resolution TEM images taken at different areas of the m$\mathrm{CoSe}_{2}$, as shown in Supplementary Fig. 21. X-ray diffraction (XRD; Fig. 2g) studies also support the phase transition of initial 
a
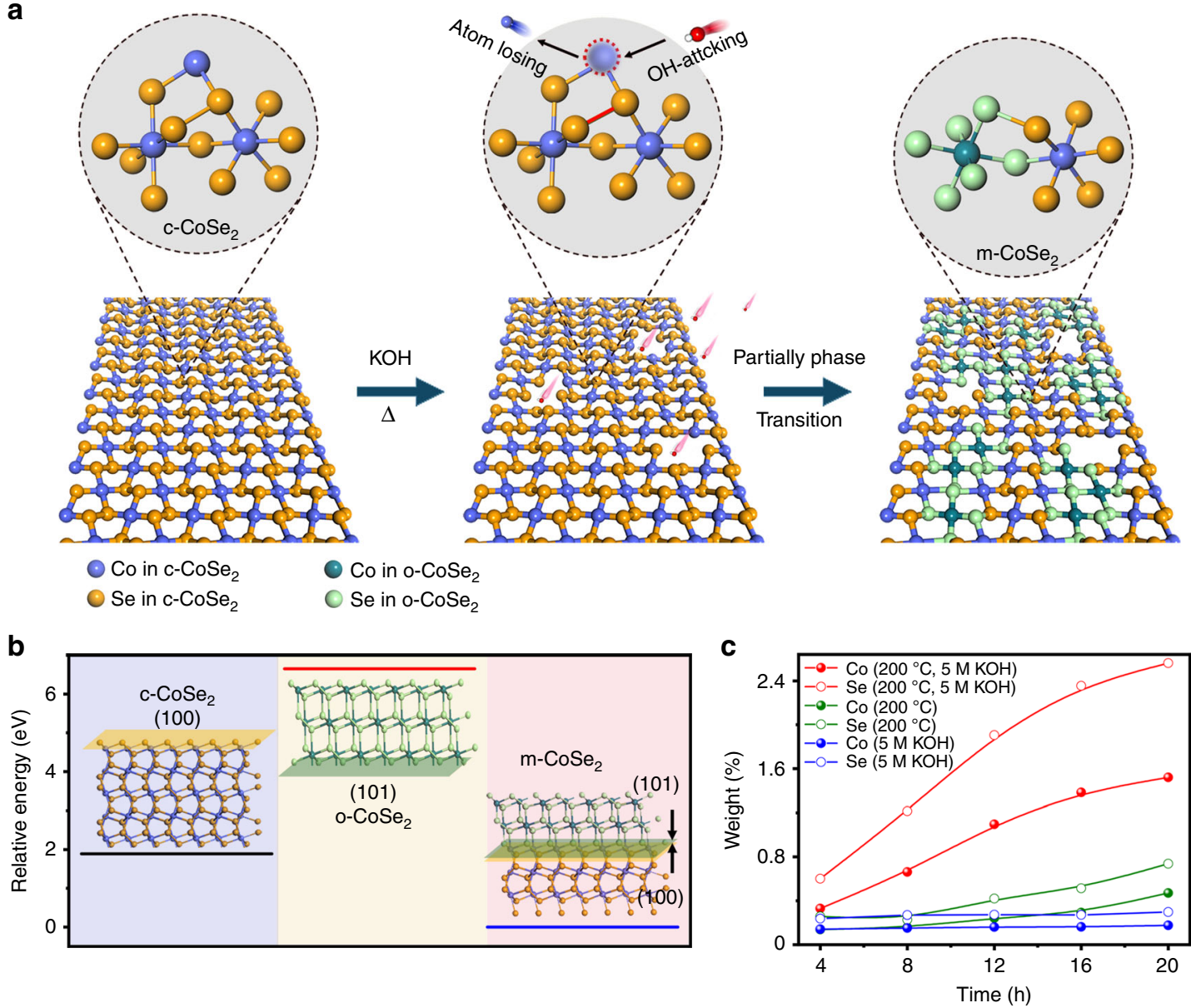

Fig. 1 Structural phase transition in c-CoSe $e_{2}$ a Schematic illustration of the defects-mediated structural phase transition from c-CoSe $e_{2}$ to $m-C_{0} e_{2}$ through

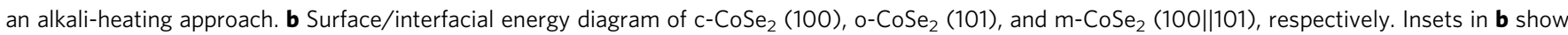
the corresponding crystal structures. c ICP-AES results compare the $\mathrm{Co}$ and Se leaching from c- $\mathrm{CoSe}_{2}$ at different reaction conditions, revealing that $\mathrm{KOH}$ and temperature are critical to drive the structural phase transition.

c- $\mathrm{CoSe}_{2}$ to phase-mixed $\mathrm{CoSe}_{2}$ (also see Supplementary Figs. 8a, $10 \mathrm{a}$ and $12 \mathrm{a})$.

Using Raman spectroscopy, we tracked the structural phase evolution in $\mathrm{c}-\mathrm{CoSe}_{2}$ (Supplementary Figs. $8 \mathrm{~b}, 10 \mathrm{~b}$ and $12 \mathrm{~b}$ ). The Raman active peak at $189 \mathrm{~cm}^{-1}$ for $\mathrm{c}-\mathrm{CoSe}_{2}$ originates from the $\mathrm{Se}-\mathrm{Se}$ stretching mode ${ }^{31}$. With tuning temperature, time or $\mathrm{KOH}$ concentration, a new Raman peak at $167 \mathrm{~cm}^{-1}$ arises gradually, indicative of the formation of orthorhombic phase. Nevertheless, Fig. $2 \mathrm{~h}$ reveals that this new peak has $\sim 7 \mathrm{~cm}^{-1}$ leftward shift compared with that of as-synthesized pure o-CoSe ${ }_{2}\left(174 \mathrm{~cm}^{-1}\right)$, suggesting the strong electronic phase interaction in $\mathrm{m}-\mathrm{CoSe}_{2}$ (ref. ${ }^{32}$ ). We also studied the work function of c-, o-, and m-CoSe by ultraviolet photoelectron spectroscopy (UPS; Fig. 2i). Our measurements display a lower work function of $\mathrm{m}-\mathrm{CoSe}_{2}(3.7 \mathrm{eV})$ compared with c-CoSe $\mathrm{C}_{2}(3.9 \mathrm{eV})$ and o- $\mathrm{CoSe}_{2}(4.6 \mathrm{eV})$, which hint at the optimized electronic structures of $\mathrm{m}-\mathrm{CoSe}_{2}$ that enable a faster electron transfer and hence superior catalytic capability.

HER activity and stability on $\mathbf{m}-\mathrm{CoSe}_{2}$ catalysts. We evaluated the electrocatalytic activities of c-, o-, and m-CoSe $\mathrm{C}_{2}$ toward HER in Ar-saturated $0.5 \mathrm{M} \mathrm{H}_{2} \mathrm{SO}_{4}$ electrolyte with Ohmic drop correction (Supplementary Fig. 22). Rotating disk electrode (RDE) testing shows that $\mathrm{m}-\mathrm{CoSe}_{2}$ requires an overpotential of mere $124 \mathrm{mV}$ at $10 \mathrm{~mA} \mathrm{~cm}^{-2}$, far exceeding that of $226 \mathrm{mV}$ for c-CoSe $\mathrm{C}_{2}$ and $273 \mathrm{mV}$ for o-CoSe $\mathrm{C}_{2}$ (Fig. 3a-c, Supplementary Fig. 22a). Tafel analysis gives a slope of 60,70 , and $37 \mathrm{mV} \mathrm{dec}^{-1}$ (here "dec" means decade, or one order of magnitude) for c-, o-, and $\mathrm{m}-\mathrm{CoSe}_{2}$ catalysts (Fig. 3b), respectively. The lower Tafel slope of $37 \mathrm{mV} \mathrm{dec}^{-1}$ for $\mathrm{m}-\mathrm{CoSe}_{2}$ indicates its HER superiority as compared to $\mathrm{c}$ - and $\mathrm{o}-\mathrm{CoSe}_{2}$ catalysts. Further, the Tafel slope of $37 \mathrm{mV} \mathrm{dec}^{-1}$ obtained for acidic HER also suggests a Tafel-stepdetermined Volmer-Tafel pathway that likely works in the $\mathrm{m}$ $\mathrm{CoSe}_{2}$ catalyst ${ }^{33} \cdot \mathrm{H}_{2}$ oxidation currents from the rotating ring-disk electrode measurements ( $\mathrm{Pt}$ ring at $0.5 \mathrm{~V}$ versus the reversible hydrogen electrode (RHE)) confirm the selective $\mathrm{H}_{2}$ production on the above catalysts (Supplementary Fig. 23). The HER performances of the $\mathrm{m}-\mathrm{CoSe}_{2}$ catalyst with different reaction temperatures, times, and $\mathrm{KOH}$ concentrations were systematically studied, as shown in Supplementary Figs. 24-26. We note that the HER kinetic metrics (e.g., $124 \mathrm{mV}$ overpotential at $10 \mathrm{~mA} \mathrm{~cm}^{-2}$ and Tafel slope of $37 \mathrm{mV} \mathrm{dec}^{-1}$ ) gained from the RDE testing ranks among the superb for noble-metal-free catalysts documented in acid ${ }^{34,35}$. The HER activity in acid of $\mathrm{m}$ $\mathrm{CoSe}_{2}$ also performs better than $\mathrm{CoSe}_{2}$ catalysts with various phases reported previously (Supplementary Fig. 27). We further detected the amount of generated $\mathrm{H}_{2}$ gas via gas chromatography, which is well consistent with the theoretical value, corresponding to a Faradaic efficiency of $99.6 \%$ (Supplementary Fig. 28). The marked HER activity of $\mathrm{m}-\mathrm{CoSe}_{2}$ can be ascribed to the large number of nanopores that offer greater accessibility to active sites, as well as the superior electronic properties originated from the unique phase-mixed structure. 



Fig. 2 Physical characterization of $\mathrm{m}-\mathrm{CoSe}_{2}$. a STEM image of a typical m-CoSe $e_{2}$ nanobelt. Scale bar, $100 \mathrm{~nm}$. b STEM-EDX elemental mapping of m-CoSe showing the homogeneous distribution of $\mathrm{Co}$ (red) and Se (yellow), respectively. Scale bar, $50 \mathrm{~nm}$. c Atomic-resolution image reconstructed by overlapping the inverse FFT patterns shown in Supplementary Fig. 18c, d. Scale bar, $5 \mathrm{~nm}$. d Atomic-resolution HAADF-STEM image of m-CoSe 2 . The regions marked by the red and yellow dashed squares indicate c-CoSe ${ }_{2}$ and o-CoSe $e_{2}$ respectively. Inset in $\mathbf{d}$ gives corresponding FFT pattern, exhibiting two sets of patterns from the [111] zone axis of $\mathrm{c}-\mathrm{CoSe}_{2}$ and [100] zone axis of o-CoSe $e_{2}$ e, $\mathbf{f}$ High-magnification HAADF-STEM images taken from the red and yellow dashed squares in $\mathbf{d}$, respectively. Scale bars, $1 \mathrm{~nm}$. Insets in $\mathbf{e}$ and $\mathbf{f}$ show the corresponding FFT patterns, evidencing the c-CoSe $e_{2}$ and o-CoSe $e_{2}$ phases, respectively. $\mathbf{g}-\mathbf{i}$ XRD patterns (g), Raman spectra (h), and UPS spectra (i) of the c-, o-, and m-CoSe ${ }_{2}$.

The $\mathrm{m}-\mathrm{CoSe}_{2}$ catalyst that survives the harsh alkali-heating conditions prompted us to carry out a comprehensive assessment of its long-term stability in acidic solution. Figure $3 \mathrm{a}-\mathrm{c}$ displays the HER polarization curves of m-, c-, and o-CoSe $e_{2}$ catalysts after different numbers of potential cycles between -0.3 and $0 \mathrm{~V}$ versus RHE, respectively. We see that the $\mathrm{m}-\mathrm{CoSe}_{2}$ catalyst requires $124 \mathrm{mV}$ overpotential at $10 \mathrm{~mA} \mathrm{~cm}^{-2}$; this value was nearly retained after 50,000 potential cycles (Fig. 3a). By comparison, c-CoSe $e_{2}$ and o-CoSe $e_{2}$ catalysts require $63 \mathrm{mV}$ (Fig. 3b) and $45 \mathrm{mV}$ (Fig. 3c) additional overpotentials at $10 \mathrm{~mA} \mathrm{~cm}^{-2}$ after mere 2000 cycles. We also performed electrochemical impedance spectroscopy (EIS) at $200 \mathrm{mV}$ overpotential to probe the charge transfer resistance $\left(R_{\mathrm{ct}}\right)$ that normalized by electrochemically active surface area (ECSA) for these catalysts. Figure $3 \mathrm{e}$ reveals that the $R_{\mathrm{ct}}$ of $\mathrm{m}-\mathrm{CoSe}_{2}$ is 1524 versus $\sim 13,660 \mathrm{ohm} \mathrm{cm} \mathrm{ECSA}^{2}$ for c-CoSe ${ }_{2}$ (Fig. 3f) and $\sim 8100$ ohm $\mathrm{cm}_{\mathrm{ECSA}}{ }^{2}$ for o-CoSe (Fig. 3g). The smallest $R_{\mathrm{ct}}$ of m-CoSe suggests its superior charge transfer kinetics, agreeing with UPS measurements. Figure 3e and Supplementary Fig. 29 also reveal that no appreciable change in $R_{\mathrm{ct}}$ was observed for $\mathrm{m}-\mathrm{CoSe}_{2}$ after 50,000 cycles, whereas the $R_{\mathrm{ct}}$ values substantially increased to $97,790 \mathrm{ohm} \mathrm{cm}_{\mathrm{ECSA}^{2}}{ }^{2}$ for c-CoSe 2 (Fig. 3f) and 22,651 ohm $\mathrm{cm}_{\mathrm{ECSA}}{ }^{2}$ for o-CoSe $\mathrm{C}_{2}$ (Fig. 3g) after 2000 cycles, consistent with results shown in Fig. 3a-c. The resistance increase hints at structural degradation of c-CoSe ${ }_{2}$ and o-CoSe $e_{2}$ in acid. Using chronopotentiometry (Fig. $3 \mathrm{~h}$ ), we found that almost no additional overpotential was required to maintain the current density of $10 \mathrm{~mA} \mathrm{~cm}^{-2}$ over $400 \mathrm{~h}$ of continuous operation in $0.5 \mathrm{M} \mathrm{H}_{2} \mathrm{SO}_{4}$, consistent with chronoamperometric measurement (Supplementary Fig. 30). All these experiments clearly demonstrate the excellent long-term catalytic stability of phase-mixed $\mathrm{CoSe}_{2}$ in acidic electrolyte. We further highlight that $\mathrm{m}-\mathrm{CoSe}_{2}$ 

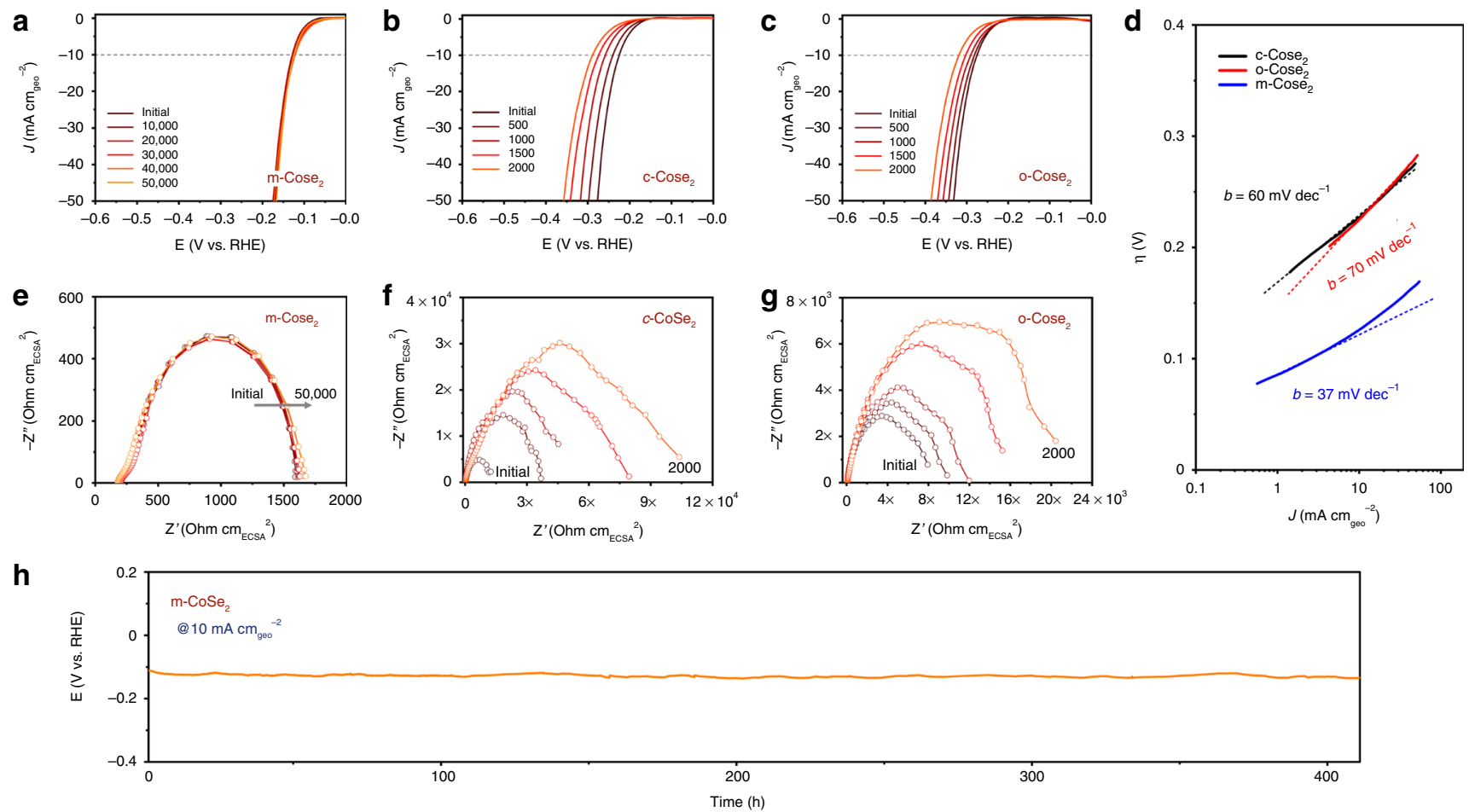

Fig. 3 Electrochemical stability of m-, c-, and o-CoSe 2 a-c HER polarization curves of m-, c-, and o-CoSe $e_{2}$ catalysts before and after different potential cycles, respectively. Catalyst loading: $1.02 \mathrm{mg} \mathrm{cm}^{-2}$. Sweep rate: $2 \mathrm{mV} \mathrm{s}^{-1}$. d Tafel plots for $\mathrm{m}-$, c-, and o-CoSe $\mathrm{C}_{2}$ catalysts derived from their initial polarization curves. e- $\mathbf{g}$ EIS Nyquist plots of the m-, c-, and o- $\mathrm{CoSe}_{2}$ catalysts before and after different potential cycles, respectively.

h Chronopotentiometry $(E \sim t)$ recorded on $\mathrm{m}-\mathrm{CoSe}_{2}$ catalyst at the constant current density of $10 \mathrm{~mA} \mathrm{~cm}^{-2}$, exhibiting the exceptional long-term stability.

catalyst can retain the HER activity even storing in laboratory for 10 months, showing its outstanding environmental stability (Supplementary Fig. 31).

Stability study of $\mathbf{m}-\mathrm{CoSe}_{2}$. We next combined multiple characterization techniques to examine the structural stability of the studied catalysts. Figure 4 a shows selective-area electron diffraction patterns of the $\mathrm{m}-\mathrm{CoSe}_{2}$ catalyst before and after 10,000, 30,000, and 50,000 potential cycles along the same zone axis. Careful analysis of these diffraction spots uncovered two sets of patterns belonging to $\mathrm{c}-\mathrm{CoSe}_{2}$ (red cycles) and $\mathrm{o}-\mathrm{CoSe}_{2}$ (yellow cycles). Notably, these diffraction peaks almost remain unchanged even after 50,000 cycles, in excellent agreement with the XRD (Fig. 4b) and Raman (Fig. 4c) results. Post-mortem TEM analysis reveals that the belt-like morphology of pristine $\mathrm{m}$-CoSe $\mathrm{Cos}_{2}$ was well retained after cycling tests (Supplementary Fig. 32). X-ray photoelectron spectra (XPS) measurements reveal Co-O $(781.09 \mathrm{eV})^{36}$ and $\mathrm{Se}-\mathrm{O}(58.59 \mathrm{eV})^{37}$ bonds for the initial $\mathrm{m}-\mathrm{CoSe}_{2}$ catalyst, which were completely removed during the durability tests (Fig. 4d, e). Furthermore, we observed that the valence states of both $\mathrm{Co}$ and Se were well maintained after 50,000 cycles, suggesting that the electronic structure of $\mathrm{m}-\mathrm{CoSe}_{2}$ was not perturbed (Fig. 4d, e, Supplementary Fig. 33). By contrast, Raman spectroscopy studies demonstrate that a noticeable peak at $248 \mathrm{~cm}^{-1}$ arises for $\mathrm{c}-\mathrm{CoSe}_{2}$ and $\mathrm{o}-\mathrm{CoSe}_{2}$ catalysts after mere 500 cycles (Supplementary Fig. 34). We attribute this new Raman active peak to the formation of amorphous Se (ref. ${ }^{38}$ ). Our ICP-AES analyses in Fig. $4 \mathrm{f}$ present severe Co and Se leaching into the electrolyte for $\mathrm{c}-\mathrm{CoSe}_{2}$ and $\mathrm{o}-\mathrm{CoSe}_{2}$, whereas the $\mathrm{m}-\mathrm{CoSe}_{2}$ catalyst almost remains physically intact (also see photographs in Supplementary Fig. 35). Additionally, we added the cycled electrolytes into the sodium acetate and nitroso- $\mathrm{R}$ salt solution, where the green solution turned to red owing to the formation of $\mathrm{Co}\left[\mathrm{C}_{10} \mathrm{H}_{4} \mathrm{ONO}\right.$ $\left.\left(\mathrm{SO}_{3} \mathrm{Na}\right)_{2}\right]_{3}$ complex ${ }^{39}$ (Fig. $4 \mathrm{~g}$ ), again showing the electrochemical leaching of $\mathrm{Co}$ for $\mathrm{c}-\mathrm{CoSe}_{2}$ and $\mathrm{o}-\mathrm{CoSe}_{2}$ samples. Together, the above results unambiguously illustrate the excellent catalytic and structural stability of the new phase-mixed $\mathrm{CoSe}_{2}$ catalyst in acidic solutions.

DFT calculation and enhancement mechanism. We now turn to discuss the structural and chemical features that potentially affect and govern the remarkable stability of $\mathrm{m}-\mathrm{CoSe}_{2}$ catalyst. We remark that cubic pyrite and orthorhombic marcasite $\mathrm{CoSe}_{2}$ are polymorphs; they both possess $\mathrm{Co}^{2+}$ occurring in octahedral coordination and contain the characteristic Se-Se pairs ${ }^{40}$ (Fig. 5a; Supplementary Fig. 36). However in cubic pyrite $\mathrm{CoSe}_{2}$, the octahedra share corners, whereas in orthorhombic marcasite they share edges $^{41}$ (Fig. 5a). Structurally, rotating half of Se-Se pairs in cubic pyrite through $90^{\circ}$ can yield the orthorhombic marcasite structure $^{40}$. The perfect lattice similarity between pyrite $\{001\}$ and marcasite $\{101\}$ allows for the epitaxial growth of $\mathrm{o}-\mathrm{CoSe}_{2}$ on c$\mathrm{CoSe}_{2}$ (Fig. 5b, inset), as confirmed by DFT calculations ${ }^{41,42}$. Although theoretically feasible, no nanostructures with a homogeneous mixture of pyrite and marcasite have been synthesized before. Here the integration of o-CoSe $e_{2}$ into $\mathrm{c}-\mathrm{CoSe}_{2}$ via harsh alkali-heating process successfully produces $\mathrm{m}-\mathrm{CoSe}_{2}$ structure, which not only brings rigid material lattices but also creates electronic structure perturbations that permit greater covalent bonding forces between $\mathrm{Co}$ and Se. Therefore, the exceptional robustness of $\mathrm{m}-\mathrm{CoSe}_{2}$ in acidic environments is expected.

To better understand the origin of the notable stability, X-ray absorption near-edge structure (XANES) spectroscopy, and DFT calculations were conducted. Figure $5 c$ gives the XANES spectra of $c-\mathrm{CoSe}_{2}$ and $\mathrm{m}-\mathrm{CoSe}_{2}$ at Co K-edge, which both show the pre- 
a

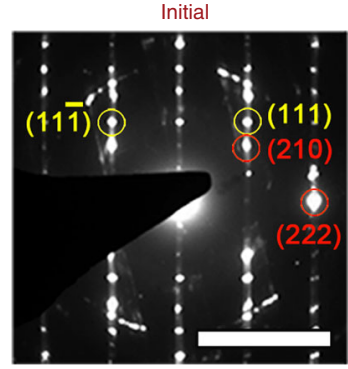

$[1 \overline{1} 1] \mathrm{c}-\mathrm{CoSe}_{2} \mathrm{ll}[1 \overline{1} \mathrm{0}] \mathrm{o}-\mathrm{CoSe}_{2}$
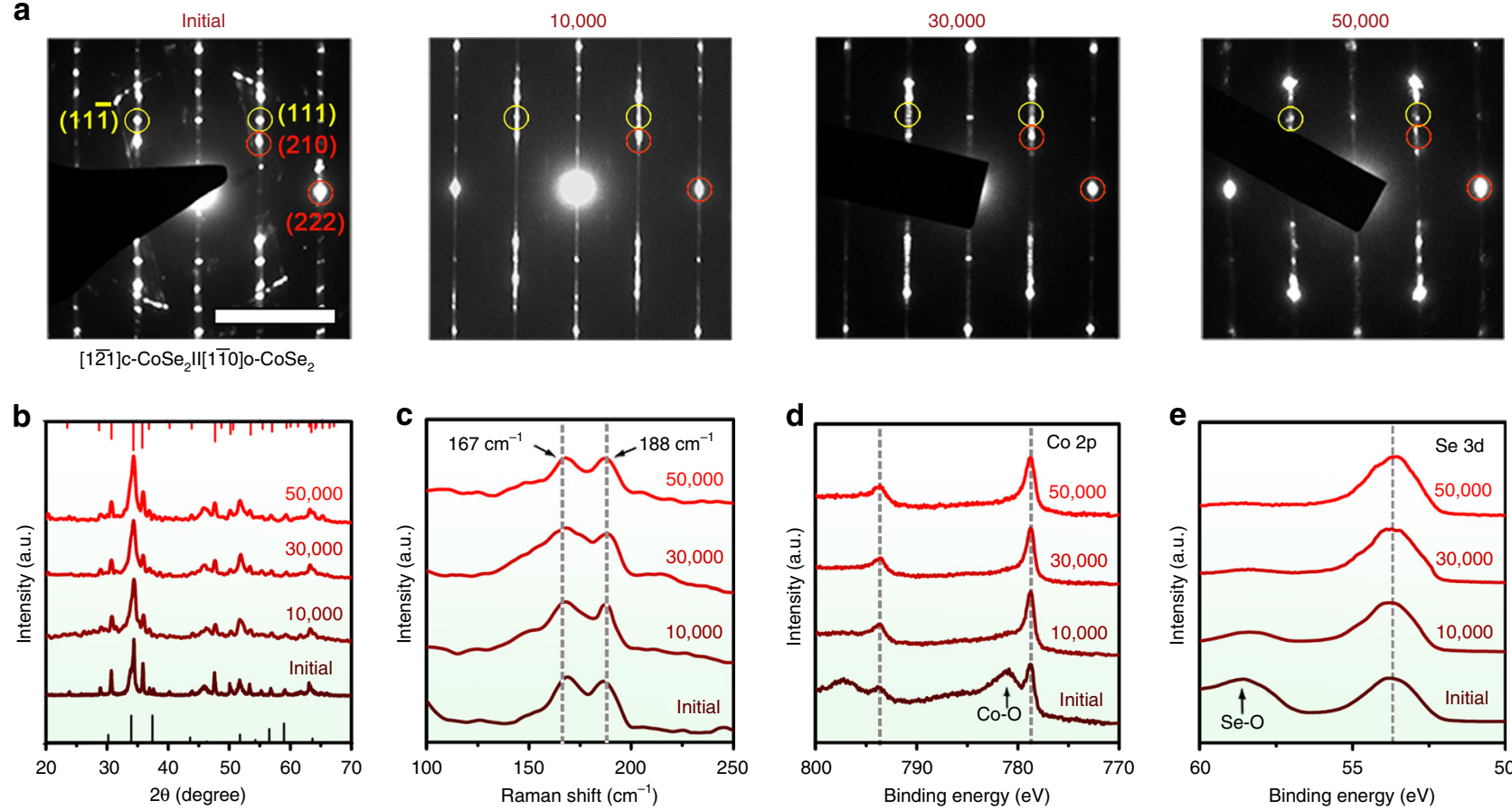

f

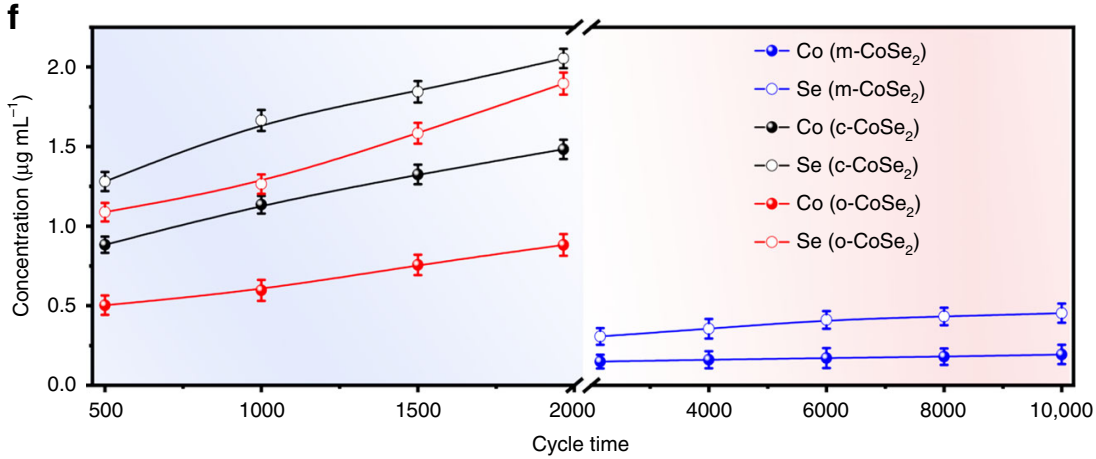

g

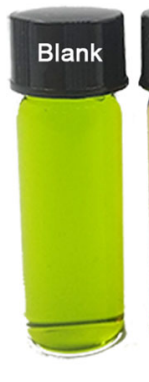

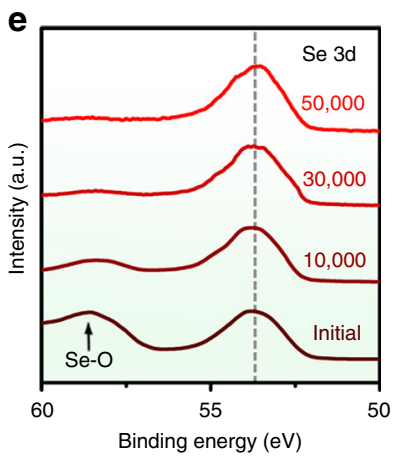
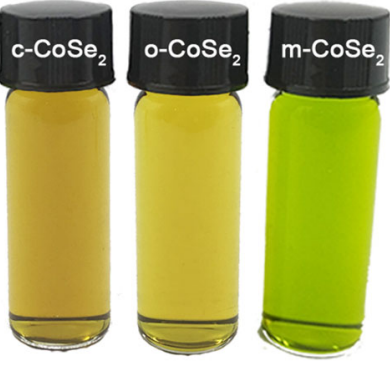

Fig. 4 Structural stability of m-CoSe 2 . a-e, SAED patterns (a), XRD patterns (b), Raman spectra (c), Co $2 p$ XPS spectra (d), and Se $3 d$ XPS spectra (e) of the $\mathrm{m}-\mathrm{CoSe}_{2}$ catalyst before and after different potential cycles. Scale bar in $\mathbf{a}, 51 / \mathrm{nm}$. f ICP-AES measurements compare the Co and Se dissolved into acidic electrolyte after different potential cycles for $\mathrm{m-}, \mathrm{c-}$, and o- $\mathrm{CoSe}_{2}$ catalysts. $\mathbf{g}$ Colorimetric comparison of the cycled electrolytes added in sodium acetate and nitroso-R salt solution. No color change was seen for $\mathrm{m}-\mathrm{CoSe}_{2}$, indicating its marked structural stability in acidic electrolyte. Nitroso-R salt was used as the color indicator. The freshly-made c- and o- $\mathrm{CoSe}_{2}$ catalysts were treated in pure deionized water at $200^{\circ} \mathrm{C}$ for $12 \mathrm{~h}$ before cycling to remove any potential unstable or amorphous components.

edge feature that indicates Co ions in the octahedral environment $^{43}$. The shoulder feature at $\sim 7717 \mathrm{eV}$ originates from the covalent nature of the Co-Se bond, analogous to previous observations in other systems ${ }^{39,44}$. The $\mathrm{m}-\mathrm{CoSe}_{2}$ catalyst reveals a considerable shift of the Co K-edge to a higher energy versus c$\mathrm{CoSe}_{2}$ (Fig. 5c), in agreement with XPS and Co L-edge measurements (Supplementary Figs. 33 and 37). This suggests a decreased occupancy of the antibonding $\mathrm{e}_{\mathrm{g}}{ }^{*}$ orbitals, pointing to a stronger Co $3 d-\mathrm{Se} 4 p$ orbital hybridization. Our Se K-edge XANES spectra shown in Fig. $5 d$ reveal that $\mathrm{m}-\mathrm{CoSe}_{2}$ exhibits a stronger peak at $12661 \mathrm{eV}$ versus c-CoSe 2 , also verifying a more covalent $\mathrm{Co}-\mathrm{Se}$ bonding orbital system ${ }^{45}$. As a result, we reason that the new phase-mixed $\mathrm{CoSe}_{2}$ catalyst shows a decreased occupancy of the antibonding $\mathrm{e}_{\mathrm{g}}^{*}$ orbitals of Co $3 d$, which tends to attract more ligand $4 p$ orbitals, enabling a greater covalency of Co-Se bond and thus substantially enhanced stability (Fig. 5e, f).

We further created the m-CoSe ${ }_{2}$ DFT model by combining the

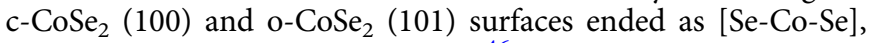
which give nonpolar terminations ${ }^{46}$ (Fig. 5b, Supplementary Figs. 38 and 39). DFT calculations show that epitaxial growth of

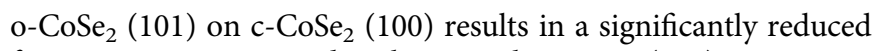
free energy as compared with original $c-\mathrm{CoSe}_{2}$ (100), suggesting that the formation of $\mathrm{m}-\mathrm{CoSe}_{2}$ is thermodynamically favorable (Fig. 5g). This accordingly implies the superior phase stability of $\mathrm{m}-\mathrm{CoSe}_{2}$. Moreover, the density of states (DOS) results reveal that the $\mathrm{m}-\mathrm{CoSe}_{2}$ catalyst exhibits lower states in the characteristic low-DOS region close to the Fermi level (Fig. 5h). The less electronic states near the Fermi level again indicate the stability of $\mathrm{m}-\mathrm{CoSe}_{2}$. Our calculations also demonstrate that the epitaxial growth of $\mathrm{o}-\mathrm{CoSe}_{2}$ on $\mathrm{c}-\mathrm{CoSe}_{2}$ is limited to a few [Se-Co-Se] layers, while further growth causes structure instability (Supplementary Figs. 40-44), agreeing with the well-distributed phase mixture of $\mathrm{m}-\mathrm{CoSe}_{2}$ we have achieved.

\section{Discussion}

A major challenge of adopting noble-metal-free catalysts in polymer electrolyte membrane-based electrochemical devices is to retain their stability in acidic environments. We have shown here that $\mathrm{m}-\mathrm{CoSe}_{2}$ is extremely stable for catalyzing HER in acid, 
a
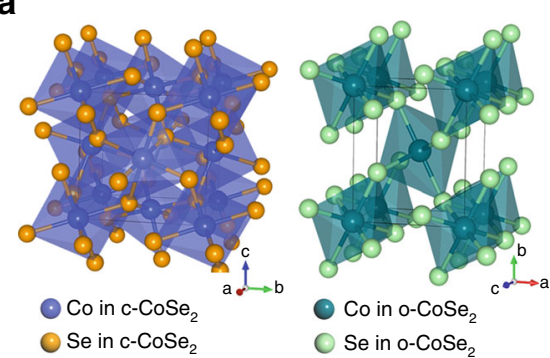

b

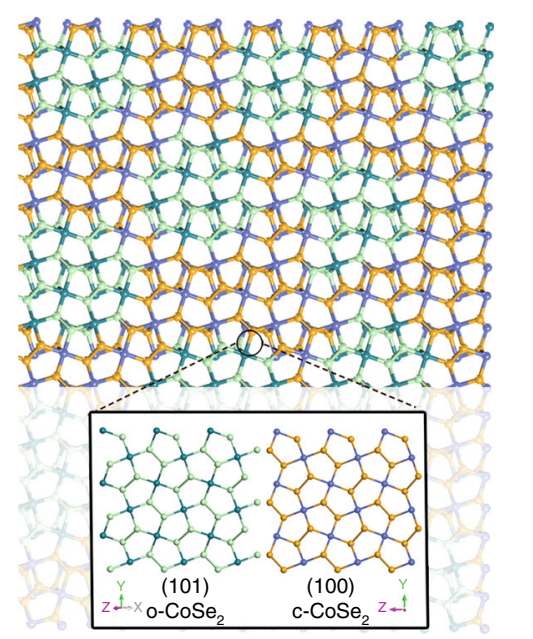

C

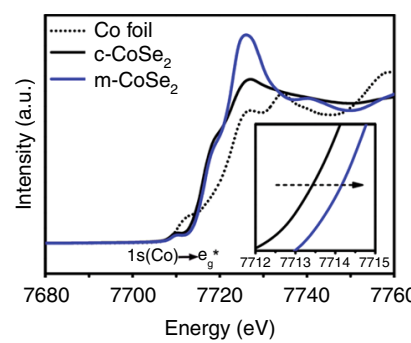

e

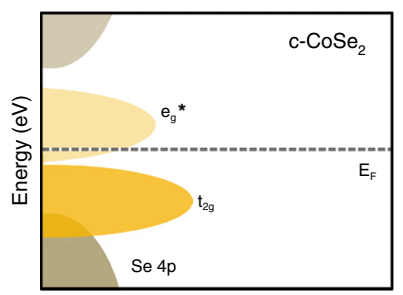

g

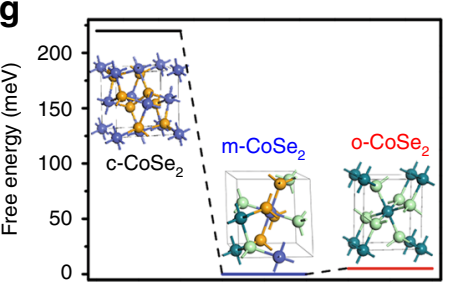

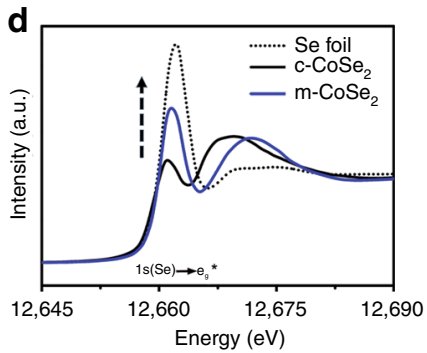

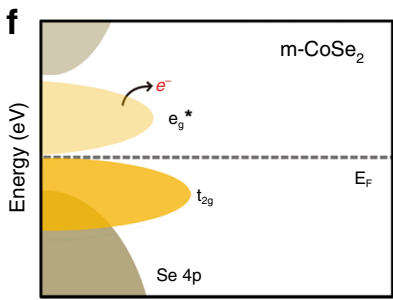

h

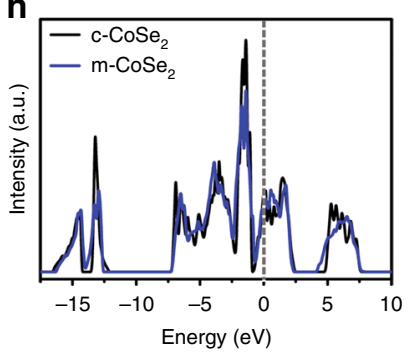

Fig. 5 DFT calculation and stability mechanism. a Crystal structures of the c-CoSe $e_{2}$ (left) and o-CoSe $e_{2}$ (right). b Schematic structure model of m-CoSe ${ }_{2}$ showing the $\left[c-\mathrm{CoSe}_{2}(100) \| \mathrm{o}-\mathrm{CoSe}_{2}(101)\right]$ interface. Insets are the atomic models of the o-CoSe $\mathrm{C}_{2}(101)$ and c-CoSe ${ }_{2}(100)$ surfaces, respectively. c, $\mathbf{d}$ XANES spectra recorded at the Co K-edge (c) and Se K-edge (d) of the Co foil, Se foil, c-CoSe $e_{2}$, and m-CoSe $e_{2}$, respectively. Inset in c shows the zoomed view of the Co K-edge spectra. e, $\mathbf{f}$ Schematic rigid band diagrams of the c- $\mathrm{CoSe}_{2}(\mathbf{e})$ and $\mathrm{m}-\mathrm{CoSe}_{2}$ (f). $\mathbf{g}$ Calculated free energy of the c-, o-, and $\mathrm{m}-\mathrm{CoSe}_{2}$ unit cells. Insets show corresponding unit cell structures. $\mathbf{h} \mathrm{DOS}$ of the $\mathrm{c}-\mathrm{CoSe}_{2}$ and $\mathrm{m}-\mathrm{CoSe}_{2}$ with the Fermi level aligned at $\mathrm{O} e \mathrm{~V}$.

whereas the stabilities of both c-CoSe $e_{2}$ and o-CoSe $e_{2}$ are ordinary. The tremendously enhanced stability of $\mathrm{m}-\mathrm{CoSe}_{2}$ can be explained by its robust lattice and the greater covalency of Co-Se bonds after phase mixing, as evidenced by multiple characterizations and DFT calculations. Given many polymorphic material systems exist, we expect that such phase-mixed engineering methodology could be intensively extended for designing cost-effective and better-performing catalysts used in acid, thus aiding the advancement of polymer electrolyte fuel cells and electrolyzers.

\section{Methods}

Material synthesis. All chemicals were used as received without further purification. The m-CoSe $e_{2}$ was synthesized through a two-step method. First, c-CoSe nanobelts were synthesized as described in our previous works ${ }^{24}$. Next, $25 \mathrm{mg} \mathrm{c}$ $\mathrm{CoSe}_{2}$ nanobelts was dispersed in $2.5 \mathrm{ml} 5 \mathrm{M} \mathrm{KOH}$ solution. After drastically stirring at room temperature for $30 \mathrm{~min}$, the black solution was transferred into a Teflon-lined autoclave $(25 \mathrm{ml})$, which was sealed and heated at $200{ }^{\circ} \mathrm{C}$ for $12 \mathrm{~h}$. After reaction, the obtained $\mathrm{m}-\mathrm{CoSe}_{2}$ powder was carefully washed and dried before use.

The o-CoSe $e_{2}$ nanobelts were synthesized by a method developed previously ${ }^{47}$. Briefly, $0.140 \mathrm{~g} \mathrm{CoSO}_{4} \cdot 7 \mathrm{H}_{2} \mathrm{O}, 0.079 \mathrm{~g} \mathrm{Se}$, and $0.180 \mathrm{~g} \mathrm{C}_{18} \mathrm{H}_{33} \mathrm{NaO}_{2}$ were added into a mixed solution $(40 \mathrm{~mL})$ with a volume ratio of $V_{\mathrm{DETA}} / V_{\mathrm{DIW}}=2: 1$ (DIW = deionzed water). After stirring for $30 \mathrm{~min}$, the mixture was transferred into a Teflon-lined autoclave and heated at $180^{\circ} \mathrm{C}$ for $30 \mathrm{~h}$. The final product was carefully washed and dried before use.

Material characterizations. The achieved samples were examined by multiple analytic techniques. XRD was taken with a Philips X'Pert Pro Super X-ray diffractometer with $\mathrm{Cu} \mathrm{Ka}$ radiation $(\lambda=1.54178 \AA)$. The morphology of the samples was investigated by SEM (Zersss Supra 40) and TEM (Hitachi H7650). The STEM and HRTEM images, SAED, and EDX elemental mappings were taken on a JEMARM 200F Atomic Resolution Analytical Microscope with an acceleration voltage of $200 \mathrm{kV}$. Raman spectra were measured on a Raman microscope $\left(\right.$ Renishaw $^{\circledR}$ ) excited with a $514 \mathrm{~nm}$ excitation laser. ICP-AES data were obtained by an Optima $7300 \mathrm{DV}$ instrument. $\mathrm{N}_{2}$ adsorption/desorption isotherms were recorded on an ASAP 2020 accelerated surface area and a porosimetry instrument (Mictromeritics), equipped with an automated surface area, at $77 \mathrm{~K}$ using Barrett-Emmett-Teller calculations. Ultraviolet photoelectron spectroscopy was carried out at the BL11U beamline of National Synchrotron Radiation Laboratory in Hefei, China. The X-ray absorption spectra of Co and Se K-edges were obtained at the beamline 14W1 of Shanghai synchrotron Radiation Laboratory (China). XPS was taken on an X-ray photoelectron spectrometer (ESCALab MKII) with an X-ray source $(\mathrm{Mg} \mathrm{Ka} h v=1253.6 \mathrm{eV})$. The X-ray absorption spectra of Co L-edges were performed on the BL10B beamline of National Synchrotron Radiation Laboratory in Hefei (China).

Electrochemical measurements. All the electrochemical measurements were measured in a conventional three-electrode cell at ambient temperature connected to a Multipotentiostat (IM6ex, ZahnerElectrik, Germany). Ag/AgCl (3.5 M KCl) electrode and graphite rod were used as the reference and counter electrodes, respectively. The potentials reported in this work were normalized versus the RHE through a standard RHE calibration described elsewhere ${ }^{48}$. A RDE with glassy carbon (PINE, $5.00 \mathrm{~mm}$ diameter, disk area: $0.196 \mathrm{~cm}^{2}$ ) was used as the working electrode.

To make the working electrodes, $5 \mathrm{mg}$ catalyst powder was dispersed in $1 \mathrm{~mL}$ of $1: 3 \mathrm{v} / \mathrm{v}$ isopropanol/DIW mixture with $40 \mu \mathrm{L}$ Nafion solution (5 wt\%), which was ultrasonicated to yield a homogeneous ink. Then, $40 \mu \mathrm{L}$ catalyst ink was pipetted onto the glassy carbon substrate (catalyst loading: $\sim 1.02 \mathrm{mg} \mathrm{cm}^{-2}$ ). HER measurements were conducted in $0.5 \mathrm{M} \mathrm{H}_{2} \mathrm{SO}_{4}$. The fresh electrolytes were bubbled with pure argon for 30 min before measurements. The polarization curves were obtained by sweeping the potential from -0.55 to $0.05 \mathrm{~V}$ versus RHE with a sweep rate of $2 \mathrm{mV} \mathrm{s}^{-1}$ and 1600 r.p.m. (to remove the $\mathrm{H}_{2}$ bubbles formed in situ) at ambient temperature. The EIS measurement was performed in the same configuration at $200 \mathrm{mV}$ overpotential over a frequency range from $100 \mathrm{KHz}$ to $100 \mathrm{mHz}$ at the amplitude of the sinusoidal voltage of $5 \mathrm{mV}$. The polarization curves were re-plotted as overpotential $(\eta)$ versus $\log$ current $(\log j)$ to get Tafel plots to assess the HER kinetics of investigated catalysts. The Tafel slope (b) can be 
obtained by fitting the linear portion of the Tafel plots with the following equation:

$$
\eta=b \log (j)+a \text {. }
$$

The $\mathrm{m}$-CoSe $\mathrm{C}_{2}$-modified carbon fiber paper (catalyst loading: $\sim 1.02 \mathrm{mg} \mathrm{cm}^{-2}$ ) was used as a working electrode to perform chronopotentiometry experiments at a constant current density of $10 \mathrm{~mA} \mathrm{~cm}^{-2}$. The accelerated stability measurements were performed by potential cycling between -0.3 and $0 \mathrm{~V}$ versus RHE with a sweep rate of $200 \mathrm{mV} \mathrm{s}^{-1}$. After cycling, the resultant electrode was used for polarization curves with a sweep rate of $2 \mathrm{mV} \mathrm{s}^{-1}$. To estimate the double-layer capacitance, cyclic voltammograms were performed at different sweep rates in the potential region of $0.0-0.1$ versus RHE at ambient temperature. All the polarization curves were corrected with $i \mathrm{R}$ compensation that resulted from the solution resistance. We employed the ICP-AES method to analyze the etching rate of $\mathrm{m}-\mathrm{CoSe}_{2}$ during chronopotentiometry experiments in $0.5 \mathrm{M} \mathrm{H}_{2} \mathrm{SO}_{4}$. The catalyst was loaded on the $1 \times 1 \mathrm{~cm}^{2}$ carbon paper substrate $\left(\sim 1.02 \mathrm{mg} \mathrm{cm}^{-2}\right)$. Each ICP-AES data point was collected for three times. The ECSA of the catalyst is calculated from the double-layer capacitance based on the equation:

$$
\mathrm{ECSA}=\frac{C_{\mathrm{dl}}}{C_{\mathrm{s}}},
$$

where $C_{\mathrm{s}}$ is the specific capacitance of the catalyst or the capacitance of an atomically smooth planar surface of the material per unit area under identical electrolyte conditions. And a general specific capacitance of $C_{\mathrm{s}}=0.035 \mathrm{mF} \mathrm{cm}-2$ is adopted based on typical reported value ${ }^{49}$.

Colorimetric comparison measurements. The $\mathrm{m}-\mathrm{CoSe}_{2}$-modified carbon fiber paper (catalyst loading: $\sim 5.10 \mathrm{mg} \mathrm{cm}^{-2}$ ) was used as a working electrode to perform accelerated stability experiments. And the electrolyte was collected after 50,000 electrochemical cycles. Then, $5 \mathrm{~mL}$ electrolyte, $5 \mathrm{~mL}$ sodium acetate solution $\left(250 \mathrm{~g} \mathrm{~L}^{-1}\right)$, and $3 \mathrm{~mL}$ of nitroso-R salt solution $\left(10 \mathrm{~g} \mathrm{~L}^{-1}\right)$ were added into a $50 \mathrm{~mL}$ beaker. The $\mathrm{Co}^{2+}$ can form a red-colored complex $\left(\mathrm{Co}\left[\mathrm{C}_{10} \mathrm{H}_{4} \mathrm{ONO}\left(\mathrm{SO}_{3} \mathrm{Na}\right)_{2}\right]_{3}\right)$ with the nitroso- $\mathrm{R}$ salt in acetate buffer solution.

DFT calculations. The DFT calculations were performed using the plane-wave code Vienna ab-initio simulation package (VASP $)^{50}$ program with the projector augmented wave $(\mathrm{PAW})^{51}$ method. The convergence criterion of the electronic self-consistent iteration was set to be $10^{-6} \mathrm{eV}$ and the kinetic energy cutoff is $400 \mathrm{eV}$. The atomic positions were relaxed until the force on each atom is below $0.005 \mathrm{eV}^{-1}$. The Perdew-Burke-Ernzerhof $(\mathrm{PBE})^{52}$ generalized gradient approximation (GGA) exchange-correlation functional was used throughout. A $(9 \times 9 \times 9)$ Monkhorst-Pack k-grid scheme was used for the calculations of c-CoSe ${ }_{2}$, $\mathrm{o}$ - $\mathrm{CoSe}_{2}$, and $\mathrm{m}-\mathrm{CoSe}_{2}$ of $N=1 ;(9 \times 9 \times 5)$ Monkhorst-Pack k-grid scheme for the $\mathrm{m}-\mathrm{CoSe}_{2}$ of $N=2$. The atomic positions and lattice constants of the pyrite, marcasite, and mixed phases are all optimized. The optimized bulk cell of pyrite is $a=$ $b=c=5.828 \AA$; the optimized lattice constants of marcasite are $\mathrm{a}=5.811 \AA$, $\mathrm{b}=$ $4.886 \AA$, and $\mathrm{c}=3.638 \AA$. Note that the $b$ constant and the [101] length $\left(\sqrt{a^{2}+c^{2}}=6.092 \AA\right)$ of marcasite are similar to the pyrite lattice constant, with lattice mismatches of $0.3 \%$ and $4.5 \%$, respectively.

\section{Data availability}

The data that support the findings of this study are available from the corresponding authors upon request.

Received: 4 June 2019; Accepted: 7 October 2019;

Published online: 25 November 2019

\section{References}

1. Dresselhaus, M. S. \& Thomas, I. L. Alternative energy technologies. Nature 414, 332 (2001).

2. Gao, M. R., Zheng, Y. R., Jiang, J. \& Yu, S. H. Pyrite-type nanomaterials for advanced electrocatalysis. Acc. Chem. Res. 50, 2194-2204 (2017).

3. Shi, Y. et al. Energy level engineering of $\mathrm{MoS}_{2}$ by transition-metal doping for accelerating hydrogen evolution reaction. J. Am. Chem. Soc. 139, 15479-15485 (2017).

4. Zhou, Y. et al. Dopant-induced electron localization drives $\mathrm{CO}_{2}$ reduction to $\mathrm{C}_{2}$ hydrocarbons. Nat. Chem. 10, 974-980 (2018).

5. $\mathrm{Li}, \mathrm{H}$. et al. Activating and optimizing $\mathrm{MoS}_{2}$ basal planes for hydrogen evolution through the formation of strained sulphur vacancies. Nat. Mater. 15, 48 (2015).

6. Wang, H. et al. Direct and continuous strain control of catalysts with tunable battery electrode materials. Science 354, 1031-1036 (2016).

7. Jia, Y., Jiang, K., Wang, H. \& Yao, X. The role of defect sites in nanomaterials for electrocatalytic energy conversion. Chem 5, 1-27 (2019).
8. Greeley, J. et al. Alloys of platinum and early transition metals as oxygen reduction electrocatalysts. Nat. Chem. 1, 552-556 (2009).

9. Escudero-Escribano, M. et al. Tuning the activity of Pt alloy electrocatalysts by means of the lanthanide contraction. Science 352, 73-76 (2016).

10. Li, J. et al. Surface evolution of a Pt-Pd-Au electrocatalyst for stable oxygen reduction. Nat. Energy 2, 17111 (2017).

11. Strasser, P. et al. Lattice-strain control of the activity in dealloyed core-shell fuel cell catalysts. Nat. Chem. 2, 454 (2010).

12. Hammer, B. \& Nørskov, J. K. Theoretical surface science and catalysiscalculations and concepts. Adv. Catal. 45, 71-129 (2000).

13. Suntivich, J., May, K. J., Gasteiger, H. A., Goodenough, J. B. \& Shao-Horn, Y A perovskite oxide optimized for oxygen evolution catalysis from molecular orbital principles. Science 334, 1383-1385 (2011).

14. Hinnemann, B. et al. Biomimetic hydrogen evolution: $\mathrm{MoS}_{2}$ nanoparticles as catalyst for hydrogen evolution. J. Am. Chem. Soc. 127, 5308-5309 (2005).

15. Su, J. et al. Assembling ultrasmall copper-doped ruthenium oxide nanocrystals into hollow porous polyhedra: highly robust electrocatalysts for oxygen evolution in acidic media. Adv. Mater. 30, 1801351 (2018).

16. Waszczuk, P. et al. Adsorption of $\mathrm{CO}$ poison on fuel cell nanoparticle electrodes from methanol solutions: a radioactive labeling study. J. Electroanal. Chem. 511, 55-64 (2001).

17. Lefèvre, M. \& Dodelet, J.-P. Fe-based catalysts for the reduction of oxygen in polymer electrolyte membrane fuel cell conditions: determination of the amount of peroxide released during electroreduction and its influence on the stability of the catalysts. Electrochim. Acta 48, 2749-2760 (2003).

18. Bi, W. \& Fuller, T. F. Modeling of PEM fuel cell Pt/C catalyst degradation. J. Power Sources 178, 188-196 (2008).

19. Arnal, P. M., Comotti, M. \& Schüth, F. High-temperature-stable catalysts by hollow sphere encapsulation. Angew. Chem. Int. Ed. 45, 8224-8227 (2006).

20. Samuels, G. J. \& Meyer, T. An electrode-supported oxidation catalyst based on ruthenium (IV). $\mathrm{pH}$ "encapsulation" in a polymer film. J. Am. Chem. Soc. 103, 307-312 (1981).

21. Wang, X. et al. Palladium-platinum core-shell icosahedra with substantially enhanced activity and durability towards oxygen reduction. Nat. Commun. $\mathbf{6}$, 7594 (2015)

22. Huang, X. et al. High-performance transition metal-doped Pt3Ni octahedra for oxygen reduction reaction. Science 348, 1230-1234 (2015).

23. Zhou, Y. et al. Enhancement of Pt and Pt-alloy fuel cell catalyst activity and durability via nitrogen-modified carbon supports. Energy Environ. Sci. 3, $1437-1446$ (2010)

24. Gao, M.-R., Yao, W.-T., Yao, H.-B. \& Yu, S.-H. Synthesis of unique ultrathin lamellar mesostructured $\mathrm{CoSe}_{2}$-amine (protonated) nanobelts in a binary solution. J. Am. Chem. Soc. 131, 7486-7487 (2009).

25. Zheng, Y. R. et al. Doping-induced structural phase transition in cobalt diselenide enables enhanced hydrogen evolution catalysis. Nat. Commun. 9, 2533 (2018)

26. Dodony, I., Pósfai, M. \& Buseck, A. R. Structural relationship between pyrite and marcasite. Am. Mineral. 81, 119-125 (1996).

27. Lin, X. et al. Intrinsically patterned two-dimensional materials for selective adsorption of molecules and nanoclusters. Nat. Mater. 16, 717-721 (2017).

28. Wang, J. \& Kaskel, S. KOH activation of carbon-based materials for energy storage. J. Mater. Chem. 22, 23710-23725 (2012).

29. Zhu, Y. et al. Carbon-based supercapacitors produced by activation of graphene. Science 332, 1537-1541 (2011).

30. Raymundo-Piñero, E. et al. $\mathrm{KOH}$ and $\mathrm{NaOH}$ activation mechanisms of multiwalled carbon nanotubes with different structural organisation. Carbon 43, 786-795 (2005).

31. Zhu, L. et al. Synthesis, characterization of a $\mathrm{CoSe}_{2}$ catalyst for the oxygen reduction reaction. Appl. Catal. A 386, 157-165 (2010).

32. Havener, R. W., Zhuang, H., Brown, L., Hennig, R. G. \& Park, J. Angleresolved Raman imaging of interlayer rotations and interactions in twisted bilayer graphene. Nano Lett. 12, 3162-3167 (2012).

33. Conway, B. \& Tilak, B. Interfacial processes involving electrocatalytic evolution and oxidation of $\mathrm{H}_{2}$, and the role of chemisorbed $\mathrm{H}$. Electrochim. Acta 47, 3571-3594 (2002).

34. Yin, Y. et al. Contributions of phase, sulfur vacancies, and edges to the hydrogen evolution reaction catalytic activity of porous molybdenum disulfide nanosheets. J. Am. Chem. Soc. 138, 7965-7972 (2016).

35. Fei, H. et al. Atomic cobalt on nitrogen-doped graphene for hydrogen generation. Nat. Commun. 6, 8668 (2015).

36. Kim, K. X-ray-photoelectron spectroscopic studies of the electronic structure of CoO. Phys. Rev. B 11, 2177 (1975).

37. Shenasa, M., Sainkar, S. \& Lichtman, D. XPS study of some selected selenium compounds. J. Electron Spectrosc. 40, 329-337 (1986).

38. Lucovsky, G., Mooradian, A., Taylor, W., Wright, G. B. \& Keezer, R. C. Identification of the fundamental vibrational modes of trigonal, a-monoclinic and amorphous selenium. Solid State Commun. 5, 113-117 (1967). 
39. Liu, W. et al. A highly active and stable hydrogen evolution catalyst based on pyrite-structured cobalt phosphosulfide. Nat. Commun. 7, 10771 (2016).

40. Tossell, J., Vaughan, D. \& Burdett, J. Pyrite, marcasite, and arsenopyrite type minerals: crystal chemical and structural principles. Phys. Chem. Miner. 7, 177-184 (1981).

41. Sun, R., Chan, M. K. Y. \& Ceder, G. First-principles electronic structure and relative stability of pyrite and marcasite: implications for photovoltaic performance. Phys. Rev. B 83, 235311 (2011).

42. $\mathrm{Wu}, \mathrm{L}$. et al. Enhanced photoresponse of $\mathrm{FeS}_{2}$ films: the role of marcasite-pyrite phase junctions. Adv. Mater. 28, 9602-9607 (2016).

43. de Groot, F., Vanko, G. \& Glatzel, P. The 1s x-ray absorption pre-edge structures in transition metal oxides. J. Phys. Condens. Matter 21, 104207 (2009).

44. Rouxel, J. Anion-cation redox competition and the formation of new compounds in highly covalent systems. Chem. Eur. J. 2, 1053-1059 (1996).

45. Sugiura, C. Sulfur $\mathrm{K} \mathrm{x}$-ray absorption spectra of $\mathrm{FeS}, \mathrm{FeS}_{2}$, and $\mathrm{Fe}_{2} \mathrm{~S}_{3}$. J. Chem. Phys. 74, 215-217 (1981).

46. Sun, R., Chan, M. K. Y., Kang, S. \& Ceder, G. Intrinsic stoichiometry and oxygen-inducedp-type conductivity of pyrite $\mathrm{FeS}_{2}$. Phys. Rev. B 84, 035212 (2011).

47. Liang, L. et al. Metallic single-unit-cell orthorhombic cobalt diselenide atomic layers: robust water-electrolysis catalysts. Angew. Chem. Int. Ed. 54, 12004-12008 (2015)

48. Li, Y. et al. $\mathrm{MoS}_{2}$ nanoparticles grown on graphene: an advanced catalyst for the hydrogen evolution reaction. J. Am. Chem. Soc. 133, 7296-7299 (2011)

49. McCrory, C. C., Jung, S., Peters, J. C. \& Jaramillo, T. F. Benchmarking heterogeneous electrocatalysts for the oxygen evolution reaction. J. Am. Chem. Soc. 135, 16977-16987 (2013).

50. Kresse, G. \& Hafner, J. Ab initiomolecular dynamics for liquid metals. Phys. Rev. B 47, 558-561 (1993).

51. Blöchl, P. E. Projector augmented-wave method. Phys. Rev. B 50, 17953-17979 (1994).

52. Perdew, J. P., Burke, K. \& Ernzerhof, M. Generalized gradient approximation made simple. Phys. Rev. Lett. 77, 3865 (1996).

\section{Acknowledgements}

This work was supported by the funding support from the National Natural Science Foundation of China (Grants 21521001, 21431006, 21225315, 21321002, 91645202, and 51702312, 21975237), the Users with Excellence and Scientific Research Grant of Hefei Science Center of CAS (2015HSCUE007), the Key Research Program of Frontier Sciences, CAS (Grant QYZDJ-SSW-SLH036), the National Basic Research Program of China (Grants 2014CB931800, 2018YFA0702001), the Chinese Academy of Sciences (Grants KGZD-EW-T05, XDA090301001), the Strategic Priority Research Program of the Chinese Academy of Sciences (XDA21000000), the Fundamental Research Funds for the Central Universities (WK2060190045, WK2340000076), and the Recruitment Program of Global Youth Experts. We would like to thank the beamline 1W1B station in the
Beijing Synchrotron Radiation Facility and BL14W1 at the Shanghai Synchrotron Radiation Facility for help with the characterizations. This work was partially carried out at the USTC Center for Micro and Nanoscale Research and Fabrication.

\section{Author contributions}

S.-H.Y. and M.-R.G. conceived and supervised the project. X.-L.Z. and Y.-R.Z. performed the experiments, collected, and analyzed the data. X.-S.Z. and J.-F.Z. performed XPS and UPS measurements. C.M. performed the HAADF-STEM measurements. S.-J.H. and X.Z. carried out the DFT calculations. R.W., F.-Y.G., P.-P.Y., Z.-Z.N., C.G. and X.-X.Y. helped with electrochemical data collection and analysis. S.-H.Y., M.-R.G. and X.-L.Z. co-wrote the manuscript. All authors discussed the results and commented on the manuscript.

\section{Competing interests}

The authors declare no competing interests.

\section{Additional information}

Supplementary information is available for this paper at https://doi.org/10.1038/s41467 019-12992-y.

Correspondence and requests for materials should be addressed to M.-R.G. or S.-H.Y.

Peer review information Nature Communications thanks the anonymous reviewers for their contributions to the peer review of this work. Peer review reports are available.

Reprints and permission information is available at http://www.nature.com/reprints

Publisher's note Springer Nature remains neutral with regard to jurisdictional claims in published maps and institutional affiliations.

\begin{abstract}
(c) (i) Open Access This article is licensed under a Creative Commons Attribution 4.0 International License, which permits use, sharing, adaptation, distribution and reproduction in any medium or format, as long as you give appropriate credit to the original author(s) and the source, provide a link to the Creative Commons license, and indicate if changes were made. The images or other third party material in this article are included in the article's Creative Commons license, unless indicated otherwise in a credit line to the material. If material is not included in the article's Creative Commons license and your intended use is not permitted by statutory regulation or exceeds the permitted use, you will need to obtain permission directly from the copyright holder. To view a copy of this license, visit http://creativecommons.org/ licenses/by/4.0\%
\end{abstract}

(c) The Author(s) 2019 